\title{
Recurrent Aseptic Basal Meningitis as the First Clinical Manifestation of a Sjogren Syndrome in a Patient with an Overlap Syndrome with Familial Mediterranean Fever
}

\author{
Milani Deb-Chatterji ${ }^{1^{*}}$, Frank Donnerstag ${ }^{2}$ and Elke V Voss ${ }^{1}$ \\ ${ }^{1}$ Department of Neurology, Hannover Medical School, Hannover, Germany \\ ${ }^{2}$ Institute of Diagnostic and Interventional Neuroradiology, Hannover Medical School, Hannover, Germany \\ ${ }^{*}$ Corresponding author: Dr. Milani Deb-Chatterji, MD, Department of Neurology, University Medical Cen- \\ ter Hamburg - Eppendorf, Hamburg, Germany, Tel: 0049-40-7410 50961; E-mail: m.deb-chatterji@uke.de
}

Received date: 31 May, 2018; Accepted date: 17 July, 2018; Published date: 01 August, 2018

\begin{abstract}
Objective: In patients suffering from Sjogren Syndrome $(\mathrm{SjS})$ central nervous system involvement, such as aseptic meningitis, commonly occurs. MRI scans of the brain usually show T2-hyperintensities and/or contrast-enhanced lesions. However, aseptic meningitis may also occur in patients with Familial Mediterranean Fever (FMF). Typical clinical symptoms are recurrent attacks of fever and polyserositis. Of note, in patients with FMF the symptoms usually disappear during a colchicine therapy.
\end{abstract}

Here, we present a patient for the first time with an overlap syndrome of $\mathrm{SjS}$ and FMF suffering from recurrent aseptic meningitis which precedes the diagnosis of SjS and shows imaging signs of a basal meningitis.

Case: A female patient suffered from biopsy-proven $\mathrm{SjS}$ and genetically diagnosed FMF. Over two years she presented with recurrent attacks of aseptic meningitis, while lacking symptoms of polyserositis indicating FMF not being causative. These attacks preceded the diagnosis of SjS. Cranial MRI revealed basal contrast enhancement of the cerebellum.

Meningitis persisted during colchicine therapy but terminated after initiating immunosuppressive treatment.

Conclusion: Recurrent aseptic meningitis may occur as the first clinical manifestation of SjS. In a basal and aseptic meningitis SjS should be considered in the differential diagnosis.

\section{Keywords}

Aseptic meningitis, Basal meningitis, Familial Mediterranean Fever, Headache, Neuroimmunology, Sjogren syndrome

\section{Introduction}

Sjogren Syndrome $(\mathrm{SjS})$ is a chronic autoimmune disease which affects exocrine glands by inflammatory infiltration and secondary dysfunction. The lacrimal and salivary glands are usually affected, thus clinically resulting in xerostomia and xerophthalmia.

Systemic extra glandular manifestations occur in one-third of all SjS patients e.g. including arthritis and vasculitis [1]. The nervous system is also often affected: the peripheral nervous system (PNS) with a frequency of $10-20 \%$ [2], including subclinical abnormalities of nerve conduction up to $60 \%$ [3], and the central nervous system (CNS) with an incidence of up to $25 \%$ [4]. Recent studies reported even higher prevalences of PNS and CNS involvements in SjS (67.5\%), with even higher rates of CNS than PNS manifestations [5]. The CNS involvement includes myelo-pathy, cortical lesions as multiple sclerosis-like syndrome, meningoencephalitis and aseptic meningitis [6].

Aseptic meningitis and other neurological symptoms are also observed in patients with Familial Mediterranean Fever (FMF). Patients with FMF suffer from recurrent attacks of fever and poly-serositis, such as pleuritis, peritonitis, synovitis/arthritis etc. [7]. While peripheral nervous system involvement is very common in FMF, CNS involvement is very rare [8].

However, recent studies revealed an incidence of $21.2 \%$ in a 
Citation: Deb-Chatterji M (2018) Recurrent Aseptic Basal Meningitis as the First Clinical Manifestation of a Sjogren Syndrome in a Patient with an Overlap Syndrome with Familial Mediterranean Fever. Curr Adv Neurol Neurol Disord 2018: 45-48. DOI: https://doi.org/10.29199/2637-6997/CANN-102021.

pediatric population [9]. In cases of a CNS affection convulsions [10], multiple sclerosis-like syndromes [11] and especially meningitis occur [12], accompanied by signs of a polyserositis. Of note, symptoms usually disappear under colchicine therapy.

Here, we report a patient with an overlap syndrome of SjS and FMF presenting with recurrent aseptic meningitis as a first clinical manifestation of SjS and showing a basal type of meningitis in the MRI for the first time. emesis, massive headache, swollen lymph nodes and splenomegaly. A MRI scan revealed no abnormalities. Initially, the symptoms were declining, but after a few days they arose again. An infection was assumed and an antibiotic therapy was initiated. After another few days the symptoms disappeared.

At the second time, the patient was admitted to hospital, she suffered again from fever accompanied by a sore throat and swollen lymph nodes. A biopsy of an affected lymph node failed to give

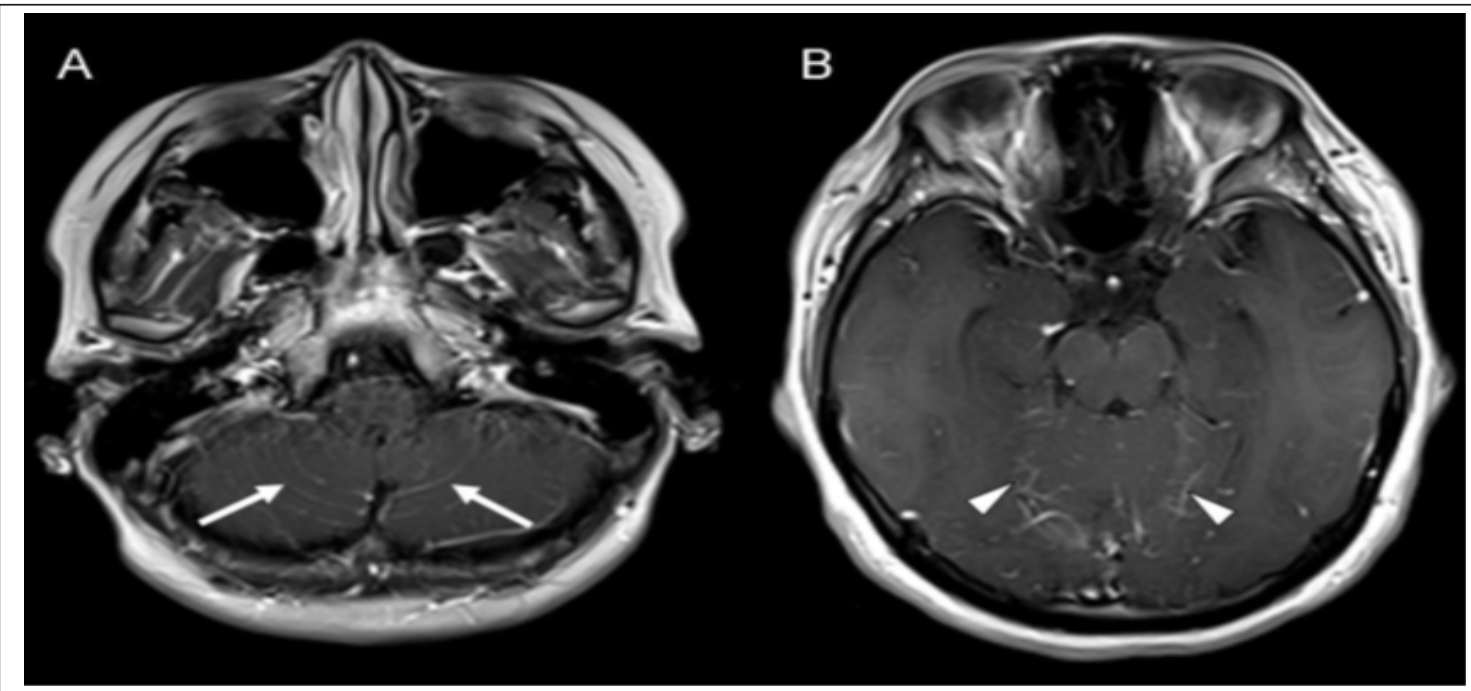

Figure 1: MRI T1 sequence after application of contrast medium (gadolinium).

A: pial gadolinium enhancement in the basal area of the cerebellum (white arrows).

B: less gadolinium enhancement in the cranial area of the cerebellum (white arrowheads).

\begin{tabular}{|c|c|c|c|c|}
\hline & 1st Episode & 2nd Episode & 3rd Episode & 4th Episode \\
\hline Cell Count $\left(/ \mu^{1}\right)$ & 54 & 88 & 210 & 228 \\
\hline Protein Level $(\mathrm{g} / \mathrm{l})$ & 0.60 & 0.70 & 0.98 & 1.36 \\
\hline Cytology & $\begin{array}{c}\text { Lymphocytic } \\
\text { predominance }\end{array}$ & $\begin{array}{c}\text { Lymphocytic } \\
\text { predominance }\end{array}$ & $\begin{array}{c}\text { Lymphocytic } \\
\text { predominance }\end{array}$ & $\begin{array}{c}\text { Lymphocytic } \\
\text { predominance }\end{array}$ \\
\hline Oligoclonal Bands & Marginal positive & Marginal positive & Identical bands & Negative \\
\hline $\begin{array}{c}\text { Evidence of Bacteri- } \\
\text { al or Viral Infection } \\
\text { (bacteriologic culture, PCR and } \\
\text { serological examinations) }\end{array}$ & Negative & Negative & Negative & Negative \\
\hline
\end{tabular}

Table 1: Results of the recurrent lumbar puncture.

(Conversion factor SI: ${ }^{1} 0.001$ )

\section{Case report}

The female patient was admitted to hospital due to recurrent attacks of fever accompanied by symptoms of polyserositis, in particular, pericarditis and peritonitis. These episodes occurred for the first time when she aged 13 .

The first attack of fever went along with a sore throat, nausea, a diagnosis. An antibiotic therapy was initiated again. Subsequently, the patient suffered from headache, nausea and emesis. A side effect of the antibiotic therapy was assumed first, but in the course of the disease the patient suffered additionally from peritonitis. A laparoscopy was performed, but an appendicitis or other pathological alterations could be excluded. After a few days the symptoms disappeared again. 
Citation: Deb-Chatterji M (2018) Recurrent Aseptic Basal Meningitis as the First Clinical Manifestation of a Sjogren Syndrome in a Patient with an Overlap Syndrome with Familial Mediterranean Fever. Curr Adv Neurol Neurol Disord 2018: 45-48. DOI: https://doi.org/10.29199/2637-6997/CANN-102021.

The third time, when the patient was admitted to hospital, she was affected by the same clinical symptoms but suffered additionally from a pericarditis. Again the symptoms declined after a few days and the patient was discharged. Finally, the diagnosis of FMF was genetically assured and an oral therapy with colchicine was initiated. After starting this medication no such episodes accompanied with symptoms of polyserositis were observed any more.

Beside these attacks the patient also suffered from recurrent episodes of fever with nausea, emesis and headache, neurological disorders like apraxia, disorientation, speech disorder and also psychiatric deficiencies, such as aggressiveness and agitation, while lacking symptoms of polyserositis. These episodes occurred four times within two years and started at an age of 17 .

The MRI scan showed a contrast enhancement of the basal regions of the cerebellum (Figure 1) at all of these timepoints.

Cerebrospinal fluid analysis revealed a pleocytosis and slightly elevated liquor protein levels without any evidence of bacterial or viral infection (Table 1).

At time of lumbar puncture the patient suffered from fever accompanied by neuropsychiatric disorders and/or headache. No systemic symptoms (e.g. polyserositis) of a FMF were observed.

Further blood examinations including immunological parameters were performed. Interestingly, positive antinuclear antibodies (ANA) and Ro-antibodies were found as an indication for $\mathrm{SjS}$ even though no typical sicca syndrome had appeared so far and the Saxon- and Schirmer-test results were recurrently negative. Finally, a salivary gland biopsy was performed that affirmed the diagnosis of a SjS. After initiation of a steroid therapy, the headache, fever and neuropsychiatric symptoms returned to normal within a few days. At the time point of the first steroid therapy the patient already received a colchicine therapy for two years.

\section{Discussion}

For the first time we report a case with recurrent aseptic meningitis which precedes the diagnosis of SjS. Additionally, it is the first time that an aseptic meningitis caused by $\mathrm{SjS}$ shows signs of a basal meningitis on the MRI scan.

In our case the patient was known to suffer from FMF and aseptic meningitis may also occur in untreated cases of FMF. However, an aseptic meningitis in FMF is usually associated with other characteristic symptoms of FMF such as polyserositis and attacks of fever $[10,12]$. These typical FMF symptoms usually disappear after initiation of a colchicine therapy $[10,12]$. A central nervous system involvement of FMF such as meningitis, accompanied headache, or multiple sclerosis - like syndromes are very unlikely under a treatment with colchicine and has not been reported so far.

Our patient showed recurrent episodes of aseptic meningitis with neuropsychiatric symptoms without signs of polyserositis despite the therapy of FMF with colchicine. Further diagnostic results with the evidence of Ro-antibodies and a typical histological finding in the salivary gland biopsy led finally to the diagnosis of SjS.

Also in SjS, multiple sclerosis - like syndromes and aseptic meningitis are documented in the literature in a few case reports $[13,14]$. However, reports with a recurrence of aseptic meningitis are rare and, to date, no such cases with signs of basal meningitis in the MRI scan have been published so far. MRI scans of the brain usually show T2-hyperintensities and/or contrast- enhanced lesions comparable to those known from multiple sclerosis. In addition, cases with a multiple cranial nerve enhancement [15], reversible cortical lesions [13], and signs of rhombencephalitis [14] were reported.

Thus, SjS must be considered in the differential diagnosis of recurrent aseptic meningitis and the diagnostic approach should include serological examinations including an immunological screening.

\section{References}

1. Haralampos M, Moutspoulos (2001). Sjoegren's syndrome. In: Harrison's Principles of Internal Medicine, (15th edn), McGraw Hill, Pg no: $1947-1949$

2. Govoni M, Padovan M, Rizzo N, Trotta F (2001) CNS involvement in primary Sjögren's syndrome: prevalence, clinical aspects, diagnostic assessment and therapeutic approach. CNS Drugs 15: 597-607.

3. Chai J, Logigian EL (2010) Neurological manifestations of primary Sjogren's syndrome. Curr Opin Neurol 23: 509-13.

4. Binder A, Snaith ML, Isenberg D (1988) Sjögren's syndrome: a study of its neurological complications. Br J Rheumatol 27: 275-80.

5. Morreale M, Marchione P, Giacomini P, Pontecorvo S, Marianetti M, et al. (2014) Neurological involvement in primary Sjögren syndrome: a focus on central nervous system. PLos One 9: e84605.

6. Rossi R, Valeria Saddi M (2006) Subacute aseptic meningitis as neurological manifestation of primary Sjögren's syndrome. Clin Neurol Neurosurg 108: 688-691.

7. Shohat M, Halpern GJ (2000) Familial Mediterranean Fever. In: Pagon RA, Adam MP, Bird TD, Dolan CR, Fong CT, Stephens K,

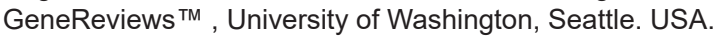

8. Finsterer J, Stöllberger C, Shinar Y (2002) Cranial nerve lesions and abnormal visually evoked potentials associated with the M694V mutation in familial Mediterranean fever. Clin Rheumatol 21: 317-321.

9. Canpolat M, Gumus H, Gunduz Z, Dusunsel R, Kumandas S, et al. (2017) Neurological Manifestations in Familial Mediterranean Fever: Results of 22 Children from a Reference Center in Kayseri, an Urban Area in Central Anatolia, Turkey. Neuropediatrics 48: 79-85.

10. Gedalia A, Zamir S (1993) Neurologic manifestations in familial Mediterranean fever. Pediatr Neurol 9: 301-302.

11. Topçuoğlu MA, Karabudak R (1997) Familial Mediterranean fever and multiple sclerosis. J Neurol 244: 510-504.

12. Karachaliou I, Karachalios G, Charalabopoulos A, Charalabopoulos K (2005) Meningitis associated with familial Mediterranean fever. Int 
Citation: Deb-Chatterji M (2018) Recurrent Aseptic Basal Meningitis as the First Clinical Manifestation of a Sjogren Syndrome in a Patient with an Overlap Syndrome with Familial Mediterranean Fever. Curr Adv Neurol Neurol Disord 2018: 45-48. DOI: https://doi.org/10.29199/2637-6997/CANN-102021.

J Clin Pract Suppl 147: 60-1.

13. Hirohata M, Yasukawa Y, Ishida C, Komai K, Yamada M (2005) Reversible cortical lesions in primary Sjögren's syndrome presenting with meningoencephalitis as an initial manifestation. J Neurol Sci 232: $111-3$
14. Chen SF, Chiu MJ, Su JJ (2011) Rhombencephalitis as an initial manifestation of primary Sjögren's syndrome: a case report and review of the literatures. Acta Neurol Taiwan 20: 35-41.

15. Sakai K, Hamaguchi T, Yamada M (2010) Multiple cranial nerve enhancement on MRI in primary Sjögren's syndrome. Intern Med 49: 857-859. 